\title{
Hacia una agricultura eficiente y sustentable
}

El mayor consumidor de agua en el planeta: la agricultura

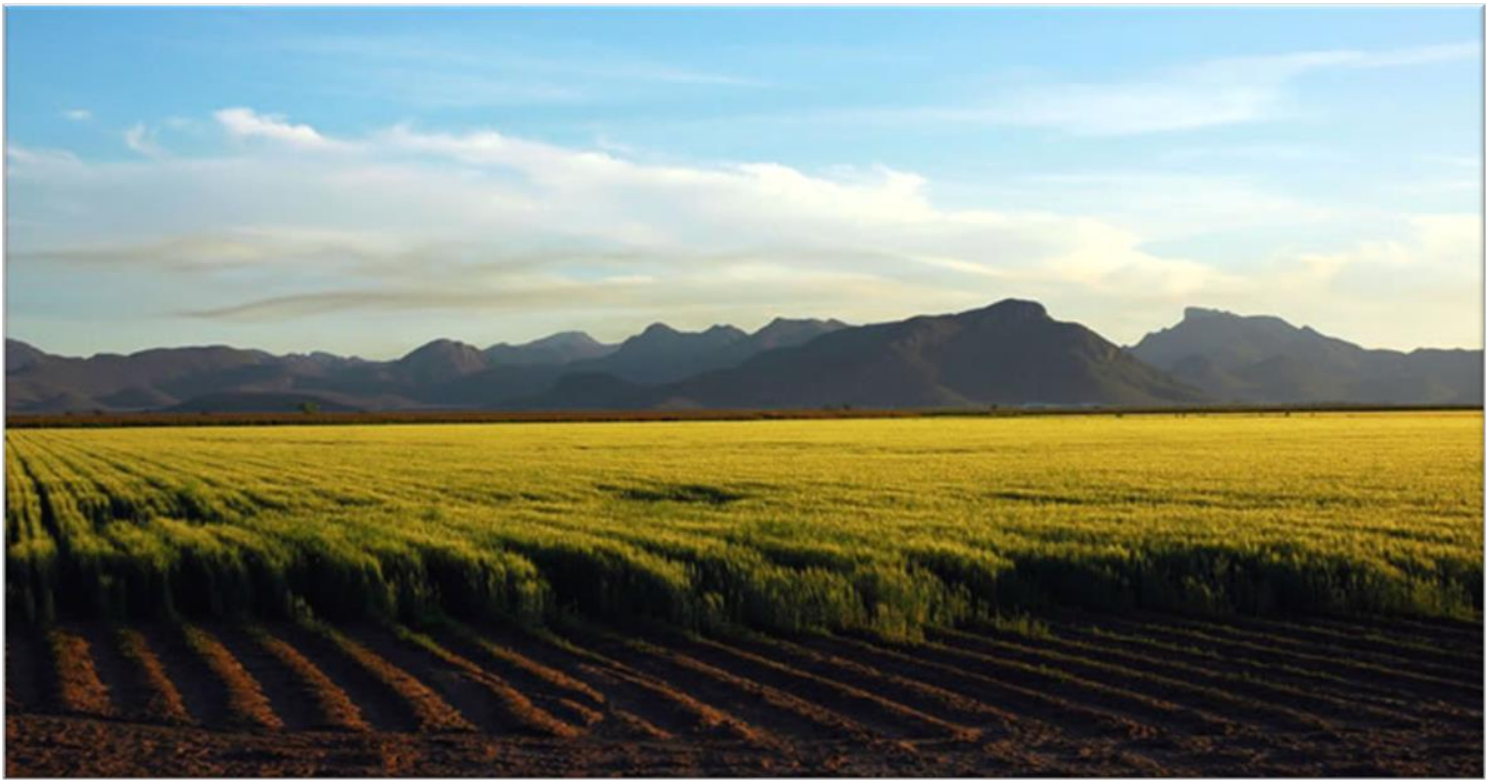

La semana pasada establecimos en este blog la posibilidad que existe en todo el mundo de llevar el consumo de agua por la industria hacia una huella hídrica cero. En esta ocasión hablaremos del mayor consumidor de agua en el planeta:

la agricultura.

En el sector agrícola no es posible aplicar el concepto de huella hídrica cero. La transpiración de las plantas es esencial para su crecimiento y para el proceso de fotosíntesis que realizan. Las estrategias de reducción de huella hídrica en la agricultura deben dirigirse, por tanto, a reducir el volumen de agua no productiva asociada con el proceso hidrológico, conocido como evapotranspiración (la suma de la evaporación y la transpiración de las plantas). Esto es posible mediante el uso de formas específicas de arado y abono de tierras (Jalota y Prihar, 1998; Nouri et al., 2019). Además, se debe considerar el uso de mejores prácticas agrícolas para incrementar los rendimientos de los cultivos por unidad de evapotranspiración. La mayoría de las veces, sobre todo en pequeños productores, los rendimientos de los cultivos suelen ser muy bajos, no como resultado del clima o del suelo, sino más bien por utilizar prácticas agrícolas rudimentarias (Molden, 2007).

La productividad hídrica se define como el cociente del rendimiento entre la evapotranspiración, y es posible incrementarla no solo reduciendo la evaporación, sino también incrementando los rendimientos. Existe un sinnúmero de medidas que un agricultor puede implementar para mejorar sus 
rendimientos, desde la mejora de la estructura del suelo y su fertilidad, hasta la selección de variedades de cultivos y patrones de siembra apropiados, lo cual requiere conocimiento detallado de la gestión agrícola y del contexto local. Por lo general no hay una solución única definitoria, sino que lo adecuado es la selección de una combinación de medidas para cada caso.

Parte de la estrategia que hemos seguido para incrementar los rendimientos agrícolas reside en el uso de sistemas de riego. Sin embargo, si estos no se diseñan y usan de forma adecuada, la evapotranspiración en los campos regados resulta mucho mayor que los rendimientos, lo que se traduce en una menor productividad hídrica. En el caso del trigo, por ejemplo, el rendimiento promedio global para zonas de riego es un tercio más grande que el de las zonas de temporal (que dependen del agua de lluvia). Sin embargo, la huella hídrica por tonelada de trigo en zonas de riego es ligeramente mayor que en zonas de temporal. Aparentemente, el riego puede dar lugar a una menor productividad hídrica, algo que suena totalmente contraintuitivo. Normalmente, la lluvia es irregular, mientras que el riego se aplica a propósito cuando se considera necesario. ¿Cómo es posible, entonces, que la productividad hídrica sea mayor en zonas de temporal que en zonas de riego? La razón de esto es que, con gran frecuencia, el riego no se realiza de forma óptima desde el punto de vista del consumo de agua.

\section{La eficiencia en el riego versus la eficiencia en el uso de agua}

Con frecuencia existe confusión entre los términos de eficiencia en el riego y la eficiencia en el uso del agua. Esto lleva a pensar que la eficiencia en el uso del agua aumenta si mejora la eficiencia en el riego. Sin embargo, este no es necesariamente el caso. El término de 'eficiencia en el riego' se refiere al porcentaje de agua extraída de un acuífero, río o lago que finalmente beneficiará a un cultivo (transpirado por la planta) (Perry, 2007). Este se define como el cociente de la transpiración del agua azul entre la cantidad total de agua utilizada en el riego (Zhuo y Hoekstra, 2017). Los ingenieros enfocan sus esfuerzos en aumentar la eficiencia del riego mediante la reducción de las pérdidas de agua en los canales de riego. Las pérdidas ocurren durante el almacenamiento, transporte, distribución y aplicación de la lámina de riego. La eficiencia promedio del sistema se define generalmente como la multiplicación de la eficiencia de conducción por la eficiencia de la aplicación de la lámina de riego. El primer término considera las pérdidas en todos los canales y conducciones hasta su aplicación en el campo y el segundo toma en cuenta la pérdida de agua posterior a la aplicación de la lámina de riego. En este sentido, la pérdida por conducción depende directamente de la longitud de los canales. Si los canales no están impermeabilizados, afecta el tipo de suelo y la permeabilidad de las márgenes, así como el estado físico de los canales y la temperatura de la región, que influye directamente en la evaporación. Típicamente, la eficiencia de conducción varía de 60 a $95 \%$, si los canales se encuentran en muy buenas condiciones (Brouwer el al., 1989). Es necesario mencionar que la falta de mantenimiento en esta infraestructura es capaz de reducir la eficiencia a la mitad. La eficiencia de aplicación en campo varía desde el 60 \% (riego por surcos) o 75 \% (riego por aspersión), hasta el 90 \% (riego por goteo). El promedio de las eficiencias en los sistemas de riego varía entre el 20 y el $85 \%$, y en algunos casos puede ser hasta del $95 \%$ si el agua es bombeada y distribuida a través de tuberías con un sistema optimizado de riego por goteo. De forma notable, el promedio global de la eficiencia en el riego se estima en el orden del 35 \% (Wallace y Gregory, 2002). Las pérdidas de agua entre la conducción y el aprovechamiento del agua por la planta o cultivo se refieren a la evaporación no benéfica (que se presenta en los embalses, canales o en el campo mismo), la filtración (en cualquier etapa) y el escurrimiento en el extremo final de los campos de cultivo.

Es importante aclarar que no es lo mismo reducir las pérdidas en el riego que reducir la huella hídrica azul de la agricultura de riego (cantidad de agua utilizada por unidad de cultivo). Dado que el concepto de huella hídrica azul se refiere a la evapotranspiración, la reducción de la pérdida en el sistema de riego es equivalente a la reducción de la huella hídrica azul solo si se refiere a la reducción de las pérdidas por 
evaporación. Los flujos de filtración y escurrimiento permanecen activos dentro de la cuenca y pueden ser reutilizados. Desde una perspectiva de cuenca, estos flujos no son considerados como pérdida. La definición de 'pérdida' que utilizan los ingenieros que diseñan los sistemas de riego es diferente a la que utilizan los administradores de una cuenca, quienes están más preocupados por uso consuntivo del agua (huella hídrica azul) dentro de la misma. El término 'eficiencia en el uso de agua' (o productividad hídrica) se refiere a la relación entre el rendimiento de un cultivo y el consumo total de agua. En general, incrementar la eficiencia de un sistema de riego por medio de la reducción de las pérdidas no benéficas por evaporación ayuda a incrementar la eficiencia en el uso de agua. Sin embargo, incrementar la eficiencia del riego a través de la reducción de las pérdidas de infiltración no tendrá el mismo efecto.

A pesar de la importante diferencia entre eficiencia de riego y eficiencia en el uso del agua, las técnicas más eficientes de riego y las estrategias de aplicación podrán también reducir considerablemente la huella hídrica azul. El uso de sistemas de riego por goteo, en lugar de sistemas de riego por aspersión o surcos, y el uso de agricultura de precisión para la aplicación de la lámina de riego tienen el potencial de reducir la evaporación sustancialmente, al mismo tiempo que se incrementan los rendimientos en los diferentes cultivos. Lo anterior, siempre y cuando los agricultores que logren una mejor eficiencia no quieran extender su superficie de cultivo.

\section{Evaluación comparativa de la huella hídrica en la agricultura}

En todo el mundo, la comparación del consumo de agua por unidad de cultivo representa una herramienta poderosa para mejorar el uso del de los recursos hídricos en la agricultura (Mekonnen y Hoekstra, 2014). Por ejemplo, se sabe que el promedio mundial de huella hídrica consuntiva para el cultivo del trigo es de 1,620 I/ kg, pero cerca del $20 \%$ de la producción global de trigo se genera con huellas hídricas consuntivas de menos de $1,000 \mathrm{l} / \mathrm{kg}$. Lo mismo ocurre en el caso del algodón, que tiene un consumo de agua promedio de 3,600 l/ $\mathrm{kg}$ por semilla de algodón, y el $20 \%$ de la producción de algodón más eficiente con una huella hídrica consuntiva de 1,820 I/ $\mathrm{kg}$ o menos. Es posible realizar un análisis como este para otros cultivos y a diferentes escalas espaciales (regiones, países o estados). La variabilidad de las huellas hídricas para cada cultivo entre regiones, países y dentro de estos nos permite establecer un análisis comparativo que define referencias respecto al consumo eficiente del agua. Adicionalmente, nos permite identificar a aquellos productores que no utilizan el agua de la manera más racional, ubicando con ello las áreas de oportunidad para la mejora en el uso de este vital líquido. Esto habilita la posibilidad de definir umbrales en el consumo de agua por cultivo que sean considerados eficientes, por ejemplo, estableciendo que los productores no excedan en su producción la huella hídrica definida por el 10-30 \% de los productores con valores menores (más eficientes). También se pueden definir de forma regional, de tal suerte que sea posible considerar las diferencias en las condiciones ambientales (clima y suelo) y de desarrollo económico de los productores. Inclusive, es posible hacer esta definición de forma global, en virtud de que para cada cultivo existe un nivel de productividad hídrica (huella hídrica) que puede ser alcanzado en cada sitio del mundo y que es adecuado para ese cultivo (Mekonnen y Hoekstra, 2014). Según Zhuo et al. (2016), si consideramos cualquier diferencia geográfica en los umbrales de huella hídrica, el factor más importante es el de la diferencia entre climas, como se demostró para el caso del trigo en China.

Otra manera de establecer los umbrales de huella hídrica por cultivo o para cualquier actividad que consuma agua es identificar la mejor tecnología disponible y tomar la huella hídrica asociada con esa tecnología como el umbral de consumo deseado (Chukalla et al., 2015). En el caso del campo, la agricultura de precisión y las técnicas de microirrigación son mucho más avanzadas que el uso de aspersores, así que se pueden utilizar como base para definir los umbrales de consumo por cultivo. Los ahorros de agua que se generen por el uso de mejores prácticas pueden ser enormes. Se sabe que, si 
PERSPECTIVAS IMTA (0)

$N^{\circ} .21,2020$

redujéramos la huella hídrica consuntiva de los cultivos en todo el mundo, para que todos los productores tuvieran a un nivel similar al $10^{\circ}$ percentil de aquellos que mejor usan el agua, el ahorro de esta sería de un 52 \% respecto al nivel actual de consumo. En el caso de huellas hídricas grises relativas al uso de nitrógeno en la producción de alimentos, si lleváramos el valor de la huella hídrica gris al nivel del $10^{\circ}$ percentil de los productores que usan menos nitrógeno, la contaminación del agua se reduciría hasta en un 79 \% (Mekonnen y Hoekstra, 2014).

El punto clave para países como el nuestro estriba en la gran desigualdad económica y disparidad social entre los pequeños y grandes agricultores, lo que necesariamente afecta su capacidad para acceder a la tecnología que puede permitir estos ahorros de agua. Por lo anterior, y por lo estratégico del agua, además de la necesidad de hacer un uso más eficiente se requiere de programas nacionales, como el de Producción para el bienestar, que estén respaldados por el mejor conocimiento disponible, el cual es generado por instituciones de investigación como el IMTA. Asimismo, además del apoyo económico que reciben estos pequeños productores, es necesario que se les brinde capacitación y tecnología de bajo costo que les permita acceder a los conocimientos y la tecnología que hagan posible estos ahorros en beneficio de toda la población mexicana.

\section{Referencias:}

Brouwer, C., Prins, K., Heibloem, M. (1989) Irrigation scheduling, Irrigation Water Management Training Manuel No. 4, Food and Agriculture Organization of the United Nations, Rome, Italy.

Chukalla, A.D., Krol, M.S., Hoekstra, A.Y., (2015) Green and blue water footprint reduction in irrigated agriculture: Effect of irrigation techniques, irrigation strategies and mulching. Hydrology and Earth System sciences, 19(12): 4877-4891.

Jalota S.K., Prohar, S.S. (1998) Reducing soil water evaporation with tillage and straw mulching, Iowa State University Press, Ames, IA, USA.

Mekonnen, M.M., Hoekstra, A.Y. (2014) Water Footprint benchmarks for crop production: A first global assessment. Ecological Indicators, 46: 214-223.

Molden, D. (ed) (2007) Water for food, water for life: A comprehensive assessment of water management in agriculture. Routledge Earthscan, London, UK.

Nouri, H., Stokvis, B., Galindo, A., Blatchford, M., Hoekstra, A.Y. (2019) Water scarcity alleviation through water footprint reduction in agriculture: The effect of soil mulching and drip irrigation, Science of the Total Environment, 653:134-139.

Perry, C. (2007) Efficient irrigation; Inefficient communication; Flawed recommendations. Irrigation and Drainage, 56(4):367-378.

Wallace, J.S.,

Gregory, P.J. (2002) Water resources and their use in food production systems. Aquatic Sciences, 64(4):363-375.

Zhuo, L., Mekonnen, M.M., Hoekstra, A.Y. (2016) The effect of inter-annual variability of consumption, production, trade and climate on crop related green and blue water footprints and inter-regional virtual water trade: A study for China - with a focus on crop production, consumption and trade. Environment International, 94:211-222. 
PERSPECTIVAS IMTA (0)

$\mathrm{N}^{\circ} .21,2020$

MEDIO AMBIENTE

(G IMTA

Autor: Adrián Pedrozo Acuña

DOI: doi.org/10.24850/b-imta-perspectivas-2020-21 